WATPHYS-TH99/02

ITP NSF-ITP-99-24

\title{
Entropy of Rotating Misner String Spacetimes
}

\author{
R.B. Mann円 \\ Institute for Theoretical Physics \\ Dept. of Physics \\ University of California Santa Barbara \\ Santa Barbara, CA USA 93106 \\ PACS numbers: 0.470.Dy, 04.20.-q
}

October 9, 2018

\begin{abstract}
Using a boundary counterterm prescription motivated by the AdS/CFT conjecture, I evaluate the energy, entropy and angular momentum of the class of Kerr-NUT/bolt-AdS spacetimes. As in the non-rotating case, when the NUT charge is nonzero the entropy is no longer equal to one-quarter of the area due to the presence of the Misner string. When the cosmological constant is also non-zero, the entropy is bounded from above.
\end{abstract}

*email: rbmann@itp.ucsb.edu

on leave from Dept. of Physics, University of Waterloo, Waterloo Ont. Canada N2L 3G1 
The thermodynamic properties of gravity have for long appeared to be inextricably connected with the presence of black holes [1,2. A physical entropy $S$ and temperature $\beta^{-1}$ can be ascribed to a given black hole configuration, where these quantities are respectively proportional to the area and surface gravity of the event horizon(s).

Recently it has been demonstrated that entropy can be associated with a broader and qualitatively different gravitational system, one containing Misner strings [3]. These objects are the gravitational analogues of Dirac strings, and arise whenever the gravitational field in the Euclidean regime has a U(1) isometry group (generated by a timelike Killing vector $\xi$ ) with a fixed point set of co-dimension $d_{f}<d-2$ (called a "nut" 㘬). The existence of any fixed point set makes it impossible to everywhere foliate the spacetime with surfaces of constant $\tau$, leading to a difference between the total energy $\left(H_{\infty}\right)$ and free energy of the gravitational system, which thermodynamically is proportional to its entropy. In general a spacetime can contain both black holes (for which $d_{f}=d-2$ - called a "bolt") and Misner strings, and the total gravitational entropy will receive contributions from both of these objects.

Explicit demonstration of these ideas has been given in a number of cases, for spacetimes with and without cosmological constant. At first it was thought that the presence of a bolt (i.e. black hole) was necessary [3,5.,6] since the Misner string contribution to the entropy is divergent - only the relative entropy (and energy) between a spacetime with a bolt/nut configuration and its asymptotically matched pure nut counterpart was calculated. However it has more recently been shown that Misner strings themselves have an intrinsic entropy [7, 8], even if no bolts are present. By adding to the action an additional boundary term which is a functional of the intrinsic curvature invariants on the boundary, the equations of motion are unaffected and the gravitational entropy (and total and free energies) is finite whether or not there is a bolt. The inclusion of this boundary term is motivated from recent work [9, 10] on the conjectured AdS/CFT duality, which equates the bulk gravitational action of an asymptotically AdS spacetime with the quantum effective action of a conformal field theory (CFT) defined on the AdS boundary. The coefficients in the additional term may be uniquely fixed by demanding that it be finite for Schwarzchild-AdS spacetime. However the spacetime need not be locally AdS asymptotically - locally asymptotically flat cases may also be included [7].

The purpose of this paper is to extend these considerations to to include rotation. Specifically I consider the class of Euclidean Kerr and Kerr-AdS solutions with and without NUT charge in four dimensions. This class of spacetimes forms an important test case for the counterterm prescription and has received relatively little attention in the literature 11, 12]. Even for Kerr spacetimes with zero nut charge and cosmological constant the problem of computing quasilocal energy is very difficult and has only been carried out in the slow-rotating limit [13]. The counterterm prescription given in ref. [7] extends to the full Kerr-NUT class, reproducing the values of the mass and angular momentum without any background spacetime subtractions. When the nut charge is non-vanishing, I find that the presence of rotation does not admit the existence of regular spacetime solutions unless a bolt is also present. Furthermore the rotation parameter has no upper bound when the nut charge and cosmological constant are nonzero. The entropies for these spacetimes are also computed and are not proportional to their horizon areas due to the Misner strings. This is the first calculation of the entropy of rotating spacetimes with nut charge. Only the main results will be presented here; details will appear in a forthcoming paper [14].

Consider a Euclidean manifold $M$ with metric $g_{\mu \nu}$, covariant derivative $\nabla_{\mu}$, and time coordinate $\tau$ which foliates $M$ into non-singular hypersurfaces $\Sigma_{\tau}$ with unit normal $u_{\mu} . \Theta^{\mu \nu}$ (whose trace is $\Theta$ ) denotes the extrinsic curvature of any boundary(ies) $\partial M$ of the manifold $M$ (internal and/or at infinity), with induced metric(s) $\gamma$. The path-integral formulation of quantum gravity implies that the Euclidean action $I=-\log Z$ to lowest order in $\hbar$, where $Z$ is the partition function of an ensemble

$$
Z=\int[D g][D \Phi] \exp [-I(g, \Phi)]
$$

with the path integral taken over all metrics $g$ and matter fields $\Phi$ that are appropriately indentified under the period $\beta$ of $\tau$. The thermodynamic definition $\log Z=S-\beta H_{\infty}$ then implies that the entropy of a given spacetime is

$$
S=\beta H_{\infty}-I=\beta(E+\Omega \cdot J)-I
$$

where $E$ and $J$ are respectively the energy and angular momentum of the spacetime at infinity and $\Omega$ the angular velocity at the event horizon. The entropy is then the difference between the value the action would have $\left(\beta H_{\infty}\right.$, the total energy) if there were no breakdown of foliation and its actual value (proportional to the free energy).

One could compute this difference by removing small neighbourhoods $N_{\epsilon}^{i}$ of the fixed point sets and strings so that $I=I_{M_{\epsilon}^{i}}-\sum_{i} I_{N_{\epsilon}^{i}}$. Rewriting the $I_{M_{\epsilon}^{i}}$ into Hamiltonian form (taking care to include the additional surface terms due to these new boundaries), one finds that the only non-zero contributions to the Hamiltonian are from the boundaries at infinity and along the strings. When the contributions $I_{N_{\epsilon}^{i}}$ from the small neighbourhoods of the fixed point sets are re-inserted, their surface terms are non-vanishing and yield the one-quarter of the areas of the neighbourhoods removed, i.e. of the bolts and the strings.

The action is generally taken to be a linear combination of a volume (or bulk) term 


$$
I_{v}=-\frac{1}{16 \pi} \int_{M} d^{d} x \sqrt{g}(R+2 \Lambda+\mathcal{L}(\Phi))
$$

and a boundary term

$$
I_{b}=-\frac{1}{8 \pi} \int_{\partial M} d^{d-1} x \sqrt{\gamma} \Theta(\gamma)
$$

(chosen to yield a well-defined variational principle), where $\mathcal{L}(\Phi)$ is the matter Lagrangian and $\Lambda$ the cosmological constant. When evaluated on solutions both $I_{v}$ and $I_{b}$ are typically divergent, yielding divergent values for both the string area and Hamiltonian terms and hence for the entropy. One method of dealing with this difficulty is to compute everything relative to some chosen reference background spacetime (suitably matched in its asymptotic and topological properties) whose boundary(ies) have the same induced metric(s) as those in the original spacetime [15 17]; the reference spacetime is then interpreted as the vacuum for that sector of the quantum theory. Such a choice is not always unique [18], nor is it always possible to embed a boundary with a given induced metric into the reference background. Indeed, for Kerr spacetimes this latter problem forms a serious obstruction towards calculating the subtraction energy, and calcuations have only been performed in the slow-rotating regime [13].

The counterterm proposal involves adding a term $I_{c t}$ to the action, where [7]

$$
I_{c t}=\frac{2}{\ell} \frac{1}{8 \pi} \int_{\partial M_{\infty}} d^{3} x \sqrt{\gamma} \sqrt{1+\frac{\ell^{2}}{2} R(\gamma)}
$$

with $\ell=\sqrt{3 /|\Lambda|}$. The coefficients of the $R(\gamma)$ term and the overall action are determined by demanding that the Schwarzchild-AdS solution have finite total action $I_{T}=I_{v}+I_{b}+I_{c t}$. The prescription (5) has been shown to be sufficient for evaluating the actions, entropies and total energies for the Schwarzchild, Taub-bolt, and Taub-NUT spacetimes, along with their AdS and topological extensions without the use of any background subtractions [7,8]. It is motivated by the conjectured AdS/CFT correspondence: divergences appearing in the stress-energy tensor of the boundary CFT are just the standard ultraviolet divergences of quantum field theory and may be removed by adding counterterms to the action which depend only on the intrinsic geometry of the boundary. Quantities such as energy, entropy and (as will be shown) angular momentum are then intrinsically defined for a given spacetime, rather than with respect to a reference background. Furthermore, (5) applies even in the $\ell \rightarrow \infty$ limit, thereby including asymptotically locally flat cases, unlike the prescriptions in refs. 83 10] to which (5) reduces for small $\ell$.

Using (5) the entropy is

$$
S=\beta H_{\infty}-\left(I_{v}+I_{b}+I_{c t}\right)
$$

where all quantities are evaluated on a given solution, and where $H_{\infty}=M+\Omega J$, with $M=Q[\partial / \partial \tau]$ and $J=Q[\partial / \partial \phi]$ being the conserved charges associated with the Killing vectors $\partial / \partial \tau$ and $\partial / \partial \phi$ where $\Omega=a /\left(r_{+}^{2}-a^{2}-N^{2}\right)$, with $r_{+}$defined below. These conserved charges are given by [16, 19

$$
Q[\xi]=\frac{1}{8 \pi} \int_{\partial M_{\infty} \cap \Sigma_{\tau}}\left[\Theta^{\mu \nu}-\Theta \gamma^{\mu \nu}+\frac{2}{\sqrt{\gamma}} \frac{\delta I_{c t}}{\gamma_{\mu \nu}}\right] u_{\mu} \xi_{\nu}
$$

which may be shown by taking the variation of the action with respect to the boundary metric $\gamma_{\mu \nu}$ at infinity .

The class of Euclidean Kerr-NUT-AdS spacetimes has the metric form

$$
\begin{aligned}
d s^{2}= & \frac{\left.V(r)\left(d \tau-\left(2 N \cos (\theta)-a \sin ^{2}(\theta)\right) d \phi\right)^{2}\right)+\mathcal{H}(\theta) \sin (\theta)^{2}\left(a d \tau-\left(r^{2}-N^{2}-a^{2}\right) d \phi\right)^{2}}{\chi^{4}\left(r^{2}-(N+a \cos (\theta))^{2}\right)} \\
& +\left(r^{2}-(N+a \cos (\theta))^{2}\right)\left(\frac{d r^{2}}{V(r)}+\frac{d \theta^{2}}{\mathcal{H}(\theta)}\right)
\end{aligned}
$$

where the Einstein field equations imply

$$
\begin{aligned}
\mathcal{H} & =1+\frac{q N^{2}}{l^{2}}+\frac{(2 N+a \cos (\theta))^{2}}{l^{2}} \\
V(r) & =\frac{r^{4}}{l^{2}}+\frac{\left((q-2) N^{2}-a^{2}+l^{2}\right) r^{2}}{l^{2}}-2 m r-\frac{(a+N)(a-N)\left(q N^{2}+l^{2}+N^{2}\right)}{l^{2}}
\end{aligned}
$$

where the periodicity in $\tau$ and the parameters $q$ and $\chi$ are chosen so that conical singularities are avoided. In the $(\theta, \phi)$ section these considerations imply that $q=-4$ and $\chi=1 / \sqrt{1+a^{2} / l^{2}}$. 
The periodicity in $\tau$ is more subtle. The location of the nut is at $r=\sqrt{a^{2}+N^{2}} \equiv r_{N}$, where the area of surfaces orthogonal to the $(r, \tau)$ section vanishes. The Misner string singularity runs along the z-axis from the nut to infinity. Regularity along the z-axis then implies that $\tau$ has period $8 \pi N$. However regularity in the $(r, \tau)$ section implies that $\tau$ also has period $2 \pi / \kappa$ where

$$
\kappa=\frac{V^{\prime}\left(r_{+}\right)}{4 \pi \chi^{2}\left(r_{+}^{2}-r_{N}^{2}\right)}
$$

where $V\left(r_{+}\right)=0, r_{+}$being the location of the foliation breakdown. Interpreting this latter equation as determining $m$ in terms of $r_{+}$, equating these two periods yields a quartic constraint (cubic if $l \rightarrow \infty$ ) on $r_{+}$in terms of $a, N$ and l. A more careful treatment is required if $r_{+}=r_{N}$; in this case regularity of the solutions demands that $V(r)$ have a double root there. However this requirement turns out to be incompatible with the periodicity constraints unless $a=0$. Hence $r_{+}>r_{N}$, and there are no regular Kerr-NUT or Kerr-NUT-AdS solutions (the former observation was made in ref. [11).

Using the formulae (7), I find after somewhat lengthy and tedious calculation

$$
M=\frac{m}{\chi^{4}} \quad J=\frac{m a}{\chi^{4}}
$$

for each of the Kerr, Kerr-AdS, Kerr-bolt and Kerr-bolt-AdS solutions, the parameter $m$ obeying the constraints mentioned in the previous paragraph in the bolt case. The actions, Hamiltonians, and entropies for each case are finite. The results are given in table I; omitted is the Hamiltonian for each case, which is simply $H_{\infty}=\frac{m}{\chi^{4}}(1+a \Omega)$. After substituing the solutions for $m$ and $r_{+}$, the action in the Kerr-bolt case is $4 \pi N m$, in agreement with ref. [11]. Note that for the bolt solutions the entropy is not one-quarter of the area, due to the presence of the Misner string.

One of the more unusual results apparent from table I is that in the Kerr-bolt-AdS solution the entropy is not positive for all values of the parameters. The entropy is always positive for any values of $a$ and $l$ provided $19 K^{6}$ $78 K^{4}+3 K^{2}+8>0$, or $K>K_{m}=2.02282556 \ldots$, where $K=r_{+} / r_{N}$. However for $1<K<K_{m}$ there exist ranges of values of $a / N$ for which the entropy is negative, and for certain values of $a$ the entropy diverges to $-\infty$. Similar properties have been noted in the non-rotating AdS NUT and bolt solutions [7,8. The entropy $s=S / N^{2}$ as a function of $x=a / N$ is plotted in Fig.1 for the Kerr-bolt solution - for small $x s \approx 5 \pi+\mathcal{O}\left(x^{2}\right)$, and for large $x, s \rightarrow 4 \pi x$. The parameter $m$ is an everywhere defined increasing function of $x$, and approaches $2 N$ for large $x$. The behaviour is quite different in the the Kerr-bolt-AdS solution. For a given $K>K_{m}, s$ reaches a maximum somewhere between $x=0$ and $x=x_{m}$; for $x>x_{m}$, the parameter $l^{2}<0$ and so there are no allowed solutions. For $K<K_{m}$, the entropy (and $m$ ) will be negative in some allowed region of $x$ (i.e. where $l^{2}>0$. Figures 2 and 3 show typical cases.

To summarize, the prescription (5) has been shown to apply to spacetimes with non-zero angular momentum, and so removes the troublesome aspects of evaluating physical quantities in gravity relative to some chosen background [13, 18. Indeed, the expressions for $E, J$ and $S$ could all be given quasilocally at finite radius $R$, although I have omitted them here for the sake of brevity. As with the non-rotating case, these results must be carefully interpreted. For $N \neq 0$ the boundary at infinity is not a direct product $S^{1} \times S^{2}$ but instead is a squashed $S^{3}$. Consequently continuation to the Lorentzian regime is not straightfoward the way it is in the $N=0$ cases. The most promising possibility is that of interpreting the path integral over all metrics as the partition function for an ensemble of spacetimes with fixed NUT charge [3,5]. A more complete understanding of the thermodynamics of these solutions (and how to interpret the negative values of the entropy in the AdS case), as well as the relationship between these results and the behaviour of a conformal field theory on the boundary remain interesting questions for further study. 


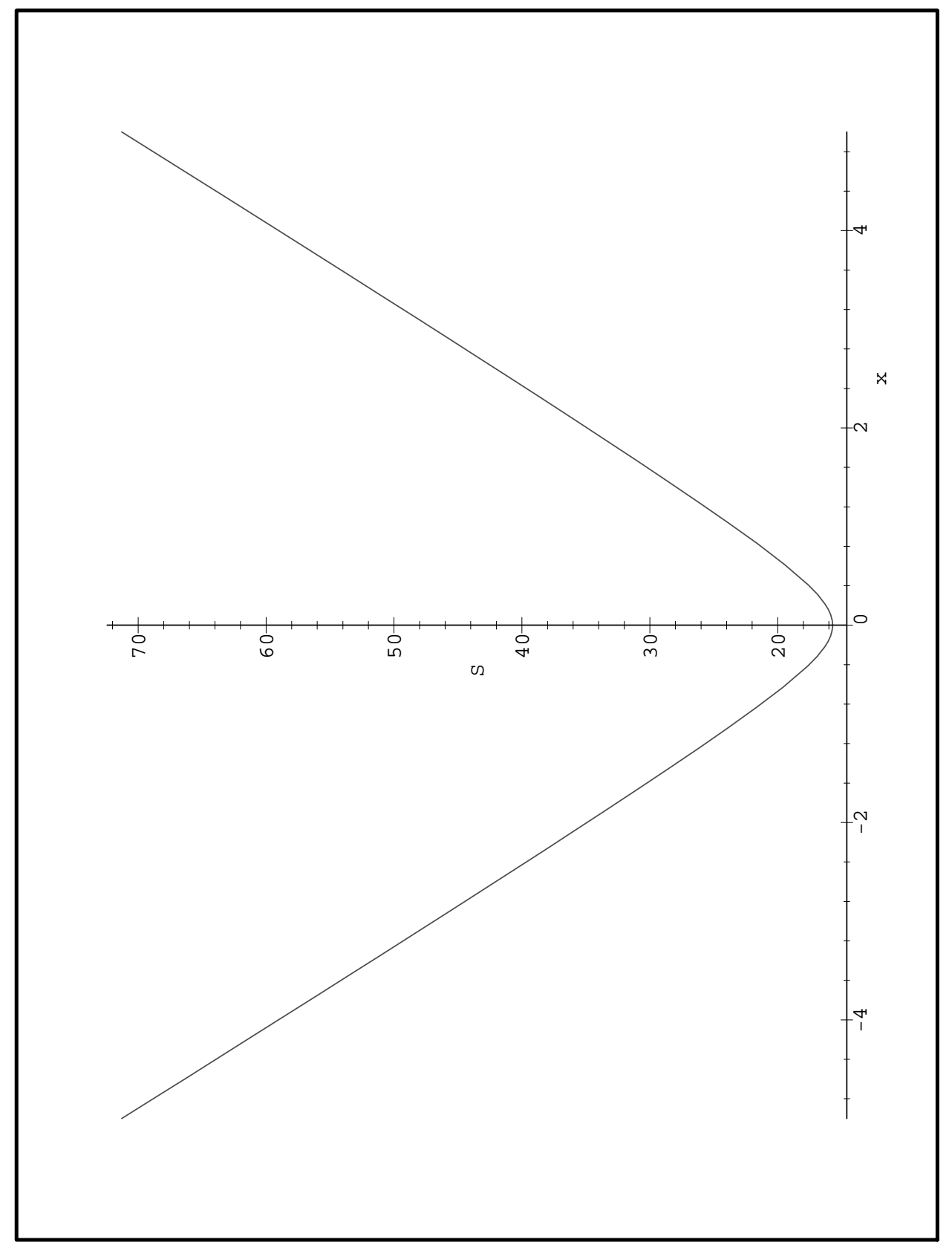

FIG. 1. The entropy as a function of $x=a / N$ in the Kerr-bolt case 


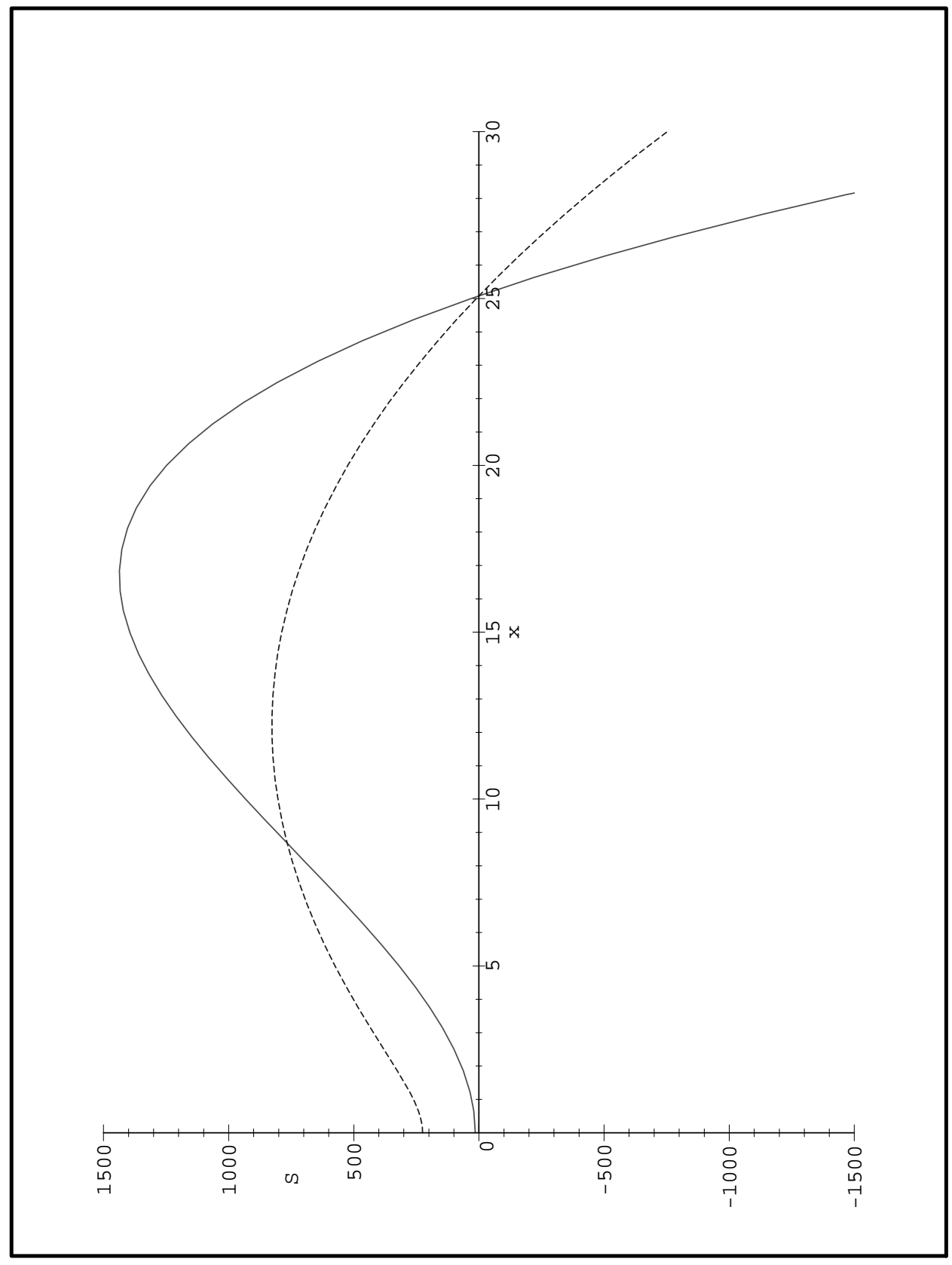

FIG. 2. The entropy (solid line) and $l^{2} / N^{2}$ (dashed line) as a function of $x=a / N$ for $K=4$ in the Kerr-bolt-AdS case. 


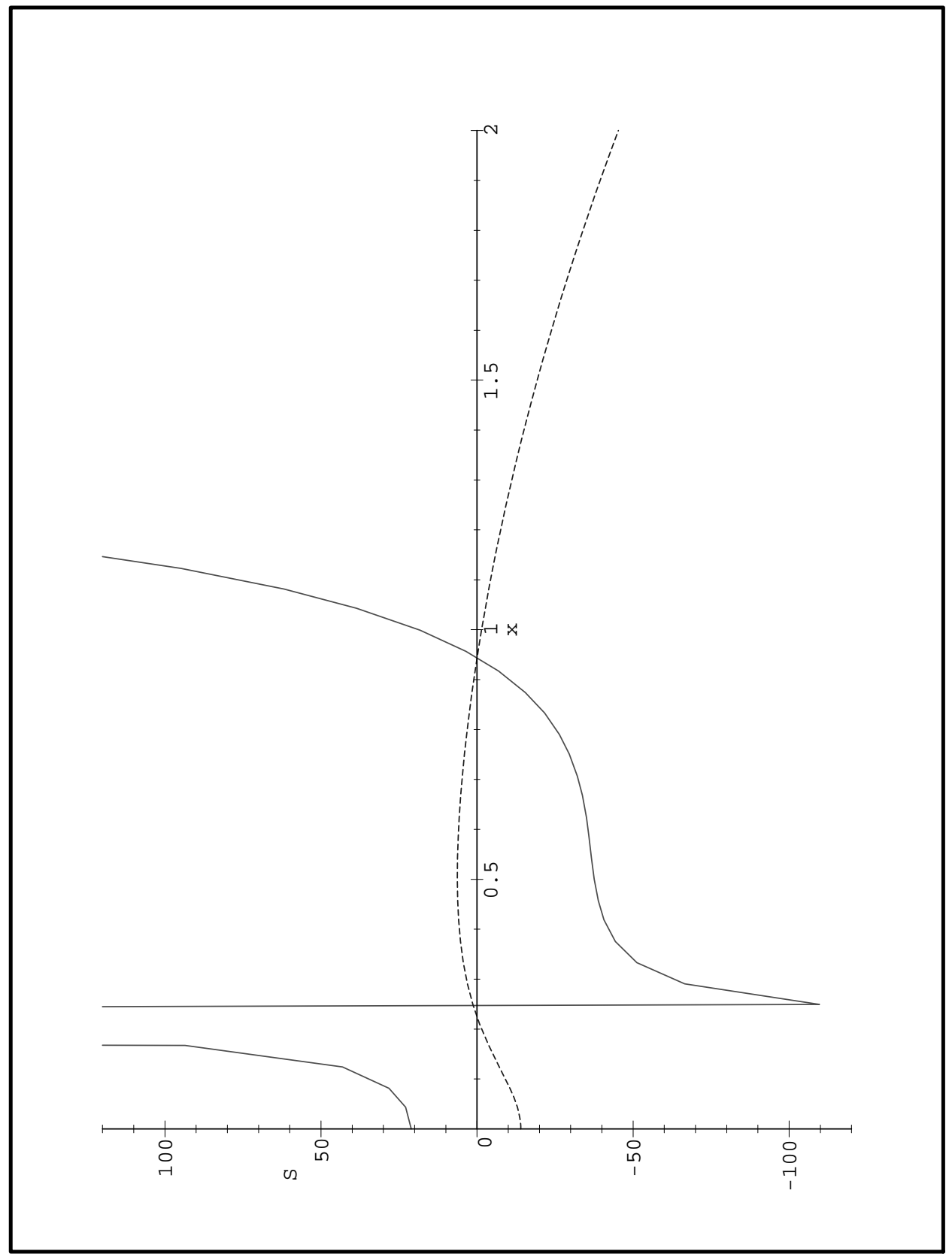

FIG. 3. The same plot as Fig. 2, but for $K=1.01$.

The work was supported by a National Science Foundation grant PHY94-07194 and by the Natural Sciences and Engineering Research Council of Canada. I am grateful to the ITP and to the physics department at UCSB for their hospitality. 
[1] J.D. Beckenstein, Phys. Rev. D7, 2333 (1973).

[2] S.W. Hawking, Nature 248, 30 (1974) ; Comm. Math. Phys. 43, 199 (1975)

[3] S.W. Hawking and C. Hunter, "Gravitational Entropy and Global Structure", hep-th 9808085.

[4] G.W. Gibbons and S.W. Hawking, Comm. Math. Phys. 66, 291 (1979).

[5] S.W. Hawking, C. Hunter and D. Page, "Nut Charge, Anti de Sitter Space and Entropy", hep-th/9809035.

[6] A. Chamblin, R. Emparan, C.V. Johnson and R. Meyers, "Large N Phases, Gravitational Instantons, and the Nuts and Bolts of AdS Holography", hep-th/9808177.

[7] R.B. Mann, "Misner String Entropy", hep-th/9903229.

[8] R. Emparan, C.V. Johnson and R. Meyers, "Surface Terms as Counterterms in the AdS/CFT Correspondence", hep-th/9903238.

[9] V. Balasubramanian and P. Kraus, "A Stress Tensor for Anti de Sitter Gravity", hep-th/9902121.

[10] S.Y. Hyun, W.T. Kim and J. Lee, Phys. Rev. D59, 084020 (1999); M. Hennigson and K. Skenderis, J.H.E.P. 9807, 23 (1998).

[11] G.W. Gibbons and M. Perry, Phys. Rev. D22, 313 (1980).

[12] A. Tomimatsu and M. Kihara, Prog. Theor. Phys. 67, 1406 (1982); M. Ahmed, Int. J. Theo. Phys. 32, 819 (1993); M. Ahmed, Phys. Lett. B258, 318 (1991).

[13] E. Martinez, Phys. Rev. D50, 4920 (1994).

[14] R.B. Mann, paper in preparation.

[15] J.D. Brown and J.W. York, Phys. Rev. D47, 1407 (1993).

[16] J.D. Brown, J. Creighton and R.B. Mann, Phys. Rev. D50, 6394 (1994).

[17] S.W. Hawking and G. Horowitz, Class. Quant. Grav. 13, 1487 (1996)

[18] K.C.K. Chan, J.D.E. Creighton, and R.B. Mann Phys. Rev. D54, 3892 (1996).

[19] I.S. Booth and R.B. Mann, Phys. Rev. D59 064010 (1999). 
Table I

\begin{tabular}{|c|c|c|c|}
\hline Spacetime & Periodicity & Action & Entropy \\
\hline Kerr & $\frac{4 \pi r_{+}\left(r_{+}^{2}-a^{2}\right)}{\left(r_{+}^{2}+a^{2}\right)}$ & $\frac{\pi\left(r_{+}^{2}-a^{2}\right)^{2}}{\left(r_{+}^{2}+a^{2}\right)}$ & $\pi\left(r_{+}^{2}-a^{2}\right)$ \\
\hline Kerr-bolt & $8 \pi N$ & $\pi \frac{\left(r_{+}^{2}-a^{2}\right)^{2}-N^{4}}{r_{+}^{2}+a^{2}-N^{2}}$ & $\pi\left(r_{+}^{2}-a^{2}+N^{2}\right)$ \\
\hline Kerr-AdS & $\frac{4 \pi r_{+}\left(\ell^{2}+a^{2}\right)\left(r_{+}^{2}-a^{2}\right)}{3 r_{+}^{4}+\left(\ell^{2}-a^{2}\right) r_{+}^{2}+a^{2} \ell^{2}}$ & $-\frac{\pi\left(r_{+}^{2}-\ell^{2}\right)\left(r_{+}^{2}-a^{2}\right)^{2}}{\left(3 r_{+}^{4}+\left(\ell^{2}-a^{2}\right) r_{+}^{2}+a^{2} \ell^{2}\right) \chi^{2}}$ & $\pi \frac{r_{+}^{2}-a^{2}}{\chi^{2}}$ \\
\hline Kerr-bolt-AdS & $8 \pi N$ & $-\pi \frac{\left(r_{+}^{2}-N^{2}-a^{2}\right)\left[r_{+}^{4}-\left(a^{2}+\ell^{2}\right) r_{+}^{2}+\left(N^{2}-a^{2}\right)\left(3 N^{2}-\ell^{2}\right)\right]}{\left(3 r_{+}^{4}+\left(\ell^{2}-a^{2}-6 N^{2}\right) r_{+}^{2}+\left(N^{2}-a^{2}\right)\left(3 N^{2}-\ell^{2}\right)\right) \chi^{2}}$ & $\pi \frac{3 r_{+}^{6}+\left(\ell^{2}-4 a^{2}-15 N^{2}\right) r_{+}^{4}+\left(a^{2}+3 N^{2}\right)^{2} r_{+}^{2}+\left(N^{2}-a^{2}\right)^{2}(3}{\left(3 r_{+}^{4}+\left(\ell^{2}-a^{2}-6 N^{2}\right) r_{+}^{2}+\left(N^{2}-a^{2}\right)\left(3 N^{2}-\ell^{2}\right)\right) \chi^{2}}$ \\
\hline
\end{tabular}

